\title{
ON THE ASYMPTOTIC DISTRIBUTION OF EIGENVALUES FOR SEMI-ELLIPTIC OPERATORS
}

\author{
BY
}

\author{
AKIRA TSUTSUMI AND CHUNG-LIE WANG(1)
}

\begin{abstract}
This paper is focused on the asymptotic distribution of eigenvalues for semielliptic operators under weaker smoothness assumptions on coefficients of operators than those of F. E. Browder [3] and Y. Kannai [8] by applying the method of Maruo-Tanabe [9].
\end{abstract}

1. Introduction and main theorem. The asymptotic behaviour of resolvent kernels and the distribution of eigenvalues for semielliptic operators have been studied by several authors, for example, F. E. Browder [3] and Y. Kannai [8]. In these works, the authors treated those operators with $C^{\infty}$ coefficients. In this note, we shall study operators having weaker smoothness conditions on their coefficients.

Let $m=\left(m_{1}, \cdots, m_{n}\right)$ and $\alpha=\left(\alpha_{1}, \cdots, \alpha_{n}\right)$ be multi-indices of positive and nonnegative integers respectively. We set

$a=1 / l$ (where $l$ indicates the least common multiple of $m_{i}$ ),

$b=\min 1 / m_{i}, c=\sum_{j=1}^{n} 1 / 2 m_{j}$, and $|\alpha: m|=\sum_{j=1}^{n} \alpha_{i} / m_{j}$.

For simplicity, the following notations will be used throughout:

(i) $D_{i}=-\sqrt{-1} \partial / \partial x_{i}$, where $D=\left(D_{1}, \cdots, D_{n}\right)$;

(ii) for a multi-index $\alpha=\left(\alpha_{1}, \cdots, \alpha_{n}\right)$ and a point $x=\left(x_{1}, \cdots, x_{n}\right)$ in a domain $\Omega$ of $R^{n}$,

$$
x^{\alpha}=x_{1}^{\alpha_{1}} \cdots x_{n}^{\alpha_{n}}, \quad D=D_{1}^{\alpha_{1}} \cdots D_{n}^{\alpha_{n}}
$$

and

$$
\begin{aligned}
& \Sigma_{(s)}=\Sigma_{|\alpha: m| \leqslant s}, \quad \Sigma_{(t)}^{\prime}=\Sigma_{|\alpha: m|=t}, \\
& \Sigma_{(s, t)}=\Sigma_{|\alpha: m| \leqslant s} \Sigma_{|\beta: m| \leqslant t}, \\
& \Sigma_{(s, t)}^{\prime}=\Sigma_{|\alpha: m|=s} \Sigma_{|\beta: m|=t} .
\end{aligned}
$$

Received by the editors June 8, 1973 and, in revised form, December 11, 1973.

AMS (MOS) subject classifications (1970). Primary 35P20, 47F05; Secondary 58 G99.

Key words and phrases. Resolvent kernels, symmetric sesquilinear form, semielliptic operators, norm inequalities, asymptotic estimates.

(1) The second author was supported (in part) by the N.R.C. of Canada (Grant No. A3116). 
A domain $\Omega$ of $R^{n}$ will be called an $n$-box with its sides of lengths $r_{i}(1 \leqslant i \leqslant n)$, if $\Omega$ is the set $\left\{x \in R^{n} \mid 0<x_{i}<r_{i}, 1 \leqslant i \leqslant n\right\}$ or its translation. Further, we denote by $H_{m}(\Omega)$ the space of functions $u$ in $L^{2}(\Omega)$ for which the distribution derivatives $D^{\alpha} u$ are also in $L^{2}(\Omega)$ for $|\alpha: m| \leqslant 1$; the space has the usual norm

$$
\|u\|_{m}=\|u\|_{m, \Omega}=\left(\int \sum_{(1)}\left|D^{\alpha} u\right|^{2} d x\right)^{1 / 2} .
$$

Finally, $\stackrel{\circ}{m}_{m}(\Omega)$ shall denote the completion of $C_{0}^{\infty}(\Omega)$ in $H_{m}(\Omega)$, where $C_{0}^{\infty}(\Omega)$ is the set of all complex valued $C^{\infty}$ functions with compact support.

Now, consider the sesquilinear form

$$
a(u, v)=\int_{\Omega} \sum_{(1,1)} a_{\alpha \beta}(x) D^{\alpha} u \overline{D^{\beta} v} d x
$$

of (multi)order $m$ and a closed subspace $V$ for which $\dot{H}_{m}(\Omega) \subset V \subset H_{m}(\Omega)$ under the following assumptions:

$$
a(u, v) \text { is symmetric: } \quad a_{\alpha \beta}(x)=\overline{a_{\alpha \beta}(x)}
$$

and

$$
a(u, v) \geqslant \delta\|u\|^{2} \quad \text { for any } u \in V,
$$

where $\delta$ is some positive constant. Studies on estimates of type (1.2) have been done by E. Giusti [6], T. Matsuzawa [10] and L. P. Volevich [15]. Associated with the sesquilinear form $a(u, v)$ is the operator $A$ on $D(A) \subset V$ to $V^{*}$ defined by $a(u, v)=(A u, v)$, for $u \in D(A)$ and for any $v \in V$. As is well known $A$ is a positive and selfadjoint operator in $L^{2}(\Omega)$. Further, the parenthesis $($,$) on the right-hand side is the duality between V^{*}$ and $V$. Identifying $L^{2}(\Omega)$ with its antidual we may consider $V \subset L^{2}(\Omega) \subset V^{*}$ algebraically and topologically. Then $V$ is a dense subspace of $V^{*}$. The following various assumptions of smoothness for the coefficients $a_{\alpha \beta}(x)$ appearing in the form $a(u, v)$ are now considered: The $a_{\alpha \beta}(x)$ are

$s$-(1) uniformly continuous for $|\alpha+\beta: m|=2$;

s-(2) uniformly Hölder continuous of order $h$ for $|\alpha+\beta: m|=2$;

s-(3) in $C^{1+h}\left(\Omega_{1}\right)$ for $|\alpha+\beta: m|=2$ and uniformly Hölder continuous of order $h$ for $2-2 a<|\alpha+\beta: m| \leqslant 2-a$;

$s$-(4) in $C^{2+h}\left(\Omega_{1}\right)$ for $|\alpha+\beta: m|=2$, in $C^{1+h}\left(\Omega_{1}\right)$ for $2-2 a<|\alpha+\beta: m| \leqslant 2-a$ and uniformly Hölder continuous of order $h$ for $2-3 a<|\alpha+\beta: m| \leqslant 2-2 a$;

$s$-(5) constants in $\Omega$ for $|\alpha+\beta: m|=2$ and in $C^{\infty}\left(\Omega_{1}\right)$ for $|\alpha+\beta: m|<2$; 
where $\Omega_{1}$ is a domain containing $\bar{\Omega}$ and $h$ is a number satisfying $0<h \leqslant a / b$.

We shall now be able to state the main theorem as follows:

THEOREM. Let $\Omega$ be an $n$-box and $c<1$. Let $N(t)$ be the number of eigenvalues of the operator. $A$ which do not exceed $t$ for $t>0$. Then the following asymptotic formulas for $N(t)$ hold as $t \rightarrow \infty$ :

$$
\begin{aligned}
& N(t)=c_{0} t^{c}+o\left(t^{c}\right) \quad \text { under } s-(1) ; \\
& N(t)=c_{0} t^{c}+O\left(t^{c-a \theta / 2}\right)
\end{aligned}
$$

for any number $\theta$ satisfying

$$
\begin{array}{ll}
0<\theta<\frac{b h}{b h+a} & \text { under s-(2); } \\
0<\theta<\frac{b(1+h)}{b(3+h)+a} & \text { under s-(3); } \\
0<\theta<\frac{b(2+h)}{b(4+h)+a} & \text { under s-(4); } \\
0<\theta<1 & \text { under s-(5); }
\end{array}
$$

where $h$ is such that $0<h \leqslant a / b$,

$$
\begin{gathered}
c_{0}=\frac{\sin (c \pi)}{c \pi} \int_{\Omega} c_{0}(x) d x, \\
c_{0}(x)=(2 \pi)^{-n} \int_{R^{n}}\left\{\sum_{(1,1)}^{\prime} a_{\alpha \beta}(x) \xi^{\alpha+\beta}+1\right\}^{-1} d \xi .
\end{gathered}
$$

2. Certain properties for operators on $H_{m}(\Omega)$. Let $Q$ be the set of rational numbers, and let

$$
\begin{aligned}
& J(m)=\{j \in Q \| \alpha: m \mid=j \text { for some multi-index } \alpha\}, \\
& J^{k}(m)=\{h \in J(m) \mid h \leqslant k\}, \quad \text { and } \\
& \|u\|_{s m}=\sum_{|\alpha: m|=s}\left\|D^{\alpha} u\right\|_{0} \text { for } s \in J(m) .
\end{aligned}
$$

A number of lemmas given on pp. 571-576 of [8] are used later; they are listed here as Lemmas 2.1-2.3.

LEMMA 2.1. If $u \in H_{s m}(\Omega)$ for any $n$-box $\Omega$ or $\Omega=R^{n}$, or if $u \in \stackrel{\circ}{s m}_{s}(\Omega)$ for any open set $\Omega$ of $R^{n}$, then $D^{\alpha} u \in L^{2}(\Omega)$ for any multiindex $\alpha$ satisfying $|\alpha: m|=s \leqslant 1$. Furthermore if the lengths of edges of an $n$-box $\Omega$ are $r_{1}, \cdots, r_{n}$, then (for $s \leqslant 1$ )

$$
\|u\|_{s m} \leqslant C r^{-\alpha}\left[\|u\|_{0}+\|u\|_{0}^{1-s}\left\{\|u\|_{m}\left(\sum_{j=1}^{n} r_{j}^{m_{j}}\right)\right\}\right],
$$

where the constant $C$ depends only on $m$. 
LEMMA 2.2 (SOBOLEV TYPE). If $u \in H_{m}(\Omega)$ for any $n$-box $\Omega$ or $\Omega=R^{n}$, or if $u \in \stackrel{H}{m}_{m}(\Omega)$ for any open set $\Omega$, then $u$ is (equal almost everywhere to) a continuous function satisfying

$$
|u(x)| \leqslant C\|u\|_{0}^{1-c / s}\|u\|_{s m}^{c / s}
$$

for a positive number $s>c$, where $C$ is a constant depending only on $m$ and on $s$.

Lemma 2.3. Let $\Omega$ be an $n$-box or $R^{n}$ and let $T$ be a bounded linear operator in $L^{2}(\Omega)$ such that the range, $R(T)$, of $T$ is contained in $H_{s m}(\Omega)$ for $s \in J(m)$ with $s>c$. Then there exists a kernel $K(x, y)$ of $T$ such that $(T f)(x)=\int_{\Omega} K(x, y) f(y) d y$ for every $f$ in $L^{2}(\Omega)$. Moreover

(i) $K\left(x_{0}, y\right) \in L^{2}(\Omega)$ for each fixed $x_{0} \in \Omega$;

(ii) for each fixed $x, K(x, \cdot)$ is a uniformly continuous function of $y$ from $\Omega$ to $L^{2}(\Omega)$

(iii) the following estimate holds:

$$
\left(\int_{\Omega}|K(x, y)|^{2} d y\right) \leqslant C\|T\|_{s m}^{c / s}\|T\|_{0}^{1-c / s},
$$

where $C$ is a constant depending only on $m$ and on $s$.

In the following setting of a bounded operator $S$ from $V^{*}$ to $V$ we use the notations

$$
\|S\|_{L^{2} \rightarrow L^{2}}, \quad\|S\|_{L^{2} \rightarrow V^{\prime}},\|S\|_{V^{*} \rightarrow V^{\prime}},\|S\|_{V^{*} \rightarrow L^{2}}
$$

to denote the norms of $S$ as an operator on $L^{2}(\Omega)$ to $L^{2}(\Omega)$, on $L^{2}(\Omega)$ to $V$, etc.

From Lemmas 2.2 and 2.3, with $s=1$, we can establish the following lemma, which is similar to Lemma 3.2 given on p. 328 of [9] (we omit its proof which is nearly identical to that of [9]).

Lemma 2.4. Let $\Omega$ be an n-box or $R^{n}$. Suppose that $S$ is a bounded linear operator on $V^{*}$ to $V$ and that $c<1$. Then there exists a function $M(x, y)$ in $C(\bar{\Omega} \times \bar{\Omega})$ such that $(S f)(x)=\int_{\Omega} M(x, y) f(y) d y$ for every $f \in L^{2}(\Omega)$. Furthermore, this kernel $M(x, y)$ of $S$ has the estimate

$$
|M(x, y)| \leqslant C\|S\|_{V^{*} \rightarrow V^{2}}^{c^{2}}\|S\|_{V^{*} \rightarrow L^{2}}^{c(1-c)}\|S\|_{L^{2} \rightarrow V^{2}}^{c(1-c)}\|S\|_{L^{2} \rightarrow L^{2}}^{(1-c)^{2}}
$$

for some constant $C$ depending only on $m$.

Let $A$ be the operator associated with the symmetric sesquilinear form $a(u, v)$ and let $\lambda$ be a complex number which is not on the positive real axis. Then by use of the Lax-Milgram theorem, we can show that the inverse, $(A-\lambda)^{-1}$, of $A-\lambda$ is a bounded operator on $V^{*}$. We now quote Lemma 3.1 given on pp. 326-327 of [9] as follows. 
Lemma 2.5. There exists a constant $C$ (depending on $\delta, \sup a_{\alpha \beta}, m$ and $n$ ) such that the following estimates hold:

$$
\begin{aligned}
& \left\|(A-\lambda)^{-1}\right\|_{L^{2} \rightarrow L^{2}} \leqslant 1 / d(\lambda), \\
& \left\|(A-\lambda)^{-1}\right\|_{L^{2} \rightarrow V} \leqslant C|\lambda|^{1 / 2} / d(\lambda), \\
& \left\|(A-\lambda)^{-1}\right\|_{V^{*} \rightarrow V} \leqslant C|\lambda| / d(\lambda), \\
& \left\|(A-\lambda)^{-1}\right\|_{V^{*} \rightarrow L^{2}} \leqslant C|\lambda|^{1 / 2} / d(\lambda) .
\end{aligned}
$$

Here and in what follows $d(\lambda)$ is defined to be the distance from the point $\lambda$ to the positive real axis.

We state, without proofs, Lemmas 2.6 and 2.7, which are similar to Lemmas 3.3 and 3.4 given on pp. 328-329 of [9].

Lemma 2.6. Let $\Omega$ be an $n$-box or $R^{n}$. Then there exists a constant $C$ (depending only on $m$ and on $s$ ) such that for any $s \in J(m)$ with $0 \leqslant s \leqslant 1$, the following estimates hold:

$$
\begin{gathered}
\left\|(A-\lambda)^{-1} f\right\|_{s m} \leqslant C|\lambda|^{1 / 2(1+s)} d(\lambda)^{-1}\|f\|_{V^{*}}, \text { for any } f \in V^{*} \text {, and } \\
\left\|(A-\lambda)^{-1} f\right\|_{s m} \leqslant C|\lambda|^{1 / 2 s} d(\lambda)^{-1}\|f\|_{0}, \text { for any } f \in L^{2}(\Omega) .
\end{gathered}
$$

LEMMA 2.7. Let $\Omega$ be an n-box or $R^{n}$. Then there exists a constant $C$ (depending only on $m$ and on $s$ ) such that for any $s \in J(m)$ with $0 \leqslant s \leqslant 1$ the estimate

$$
\|u\|_{s m} \leqslant C|\lambda|^{-1 / 2(1-s)}\left(\|u\|_{m}+|\lambda|^{1 / 2}\|u\|_{0}\right)
$$

holds for any $u \in V$.

3. Estimates of the difference between resolvent kernels on $\dot{H}_{m}(\Omega)$ and $V$. An operator $A: V \rightarrow V^{*}$ is defined by $a(u, v)=(A u, v)$ for any $u, v \in V$ while an operator $A_{0}: \stackrel{\circ}{H}_{m}(\Omega) \rightarrow H_{-m}(\Omega)$ is defined by $a(u, v)=\left(A_{0} u, v\right)$ for any $u, v \in \stackrel{\circ}{H}_{m}(\Omega)$. Although both operators $A$ and $A_{0}$ are associated with the symmetric sesquilinear form $a(u, v)$, the parenthesis $(A u, v)$ denotes the duality between $V^{*}$ and $V$ in the former and the parenthesis $\left(A_{0} u, v\right)$, the duality between $H_{-m}(\Omega)$ (the anti-dual of $\dot{H}_{m}(\Omega)$ ) and $\dot{H}_{m}(\Omega)$ in the latter. Evidently, for the operator $A_{0}$ the analogues of Lemmas 2.5-2.7 hold. Let

$$
\Lambda=\left\{g \in C_{0}^{\infty}(U) \mid g(0)=1\right\},
$$

where $U=\left\{x \in R^{n}|| x_{i} \mid<1,1 \leqslant i \leqslant n\right\}$. Let $x^{0}$ be a fixed point in $\Omega$. For simplicity, we put $\epsilon=\delta\left(x^{0}\right)$ and 


$$
\eta_{\epsilon}(x)=\eta\left(\frac{x_{1}-x_{1}^{0}}{\epsilon^{1 / m_{1}}}, \cdots, \frac{x_{n}-x_{n}^{0}}{\epsilon^{1 / m_{n}}}\right)=\eta\left(\frac{x-x^{0}}{\epsilon^{(1 / m)}}\right)
$$

for $\eta \in \Lambda$. Note that $\operatorname{Sup}_{x \in \Omega}\left|D^{\alpha} \eta_{\epsilon}(x)\right| \leqslant C \epsilon^{-|\alpha: m|}$ holds, where $C$ is a constant depending only on $m$ and on $\eta$. For any $f \in V^{*}$ we denote $f \mid \dot{H}_{m}(\Omega)$ by $r f$. Note also that $(r f, \phi)=(f, \phi)$ for $\phi \in H_{m}(\Omega) \subset V$. Using the mappings

$$
(A-\lambda)^{-1}: V^{*} \rightarrow V \text { and }\left(A_{0}-\lambda\right)^{-1}: H_{-m}(\Omega) \rightarrow \stackrel{\circ}{H}_{m}(\Omega),
$$

the operator $S_{\lambda_{\epsilon}}: V^{*} \rightarrow \dot{H}_{m}(\Omega)$ can be defined by

$$
S_{\lambda_{\epsilon}} f=\eta_{\epsilon}\left\{(A-\lambda)^{-1} f-\left(A_{0}-\lambda\right)^{-1}(r f)\right\}
$$

for any $f \in V^{*}$. Obviously, $S_{\lambda_{\epsilon}}$ is a bounded operator on $V^{*}$ to $\dot{H}_{m}(\Omega)$ and hence a fortiori to $V$.

LEMMA 3.1. If $\epsilon^{-1}|\lambda|^{-1 / 2} \leqslant 1$ and $|\lambda| \geqslant 1$, then for any positive integer $j$ there exists a positive constant $K_{j}$ such that

$$
\left\|S_{\lambda_{\epsilon}}\right\|_{V^{*} \rightarrow V} \leqslant K_{j} F(\epsilon, \lambda, j)|\lambda| d(\lambda)^{-1},
$$

$$
\left\|S_{\lambda_{\epsilon}}\right\|_{V^{*} \rightarrow L^{2}, L^{2} \rightarrow V} \leqslant K_{j} F(\epsilon, \lambda, j)|\lambda|^{1 / 2} d(\lambda)^{-1} \text {, }
$$

$$
\left\|S_{\lambda_{\epsilon}}\right\|_{L^{2} \rightarrow L^{2}} \leqslant K_{j} F(\epsilon, \lambda, j) d(\lambda)^{-1} \text {, }
$$

where $F(\epsilon, \lambda, j)=\left(\epsilon^{-b}|\lambda|^{1-b / 2} d(\lambda)^{-1}\right)^{j}$.

Proof. Let $u=(A-\lambda)^{-1} f-\left(A_{0}-\lambda\right)^{-1}(r f)$ and $v=\eta_{\epsilon} u=S_{\lambda_{\epsilon}} f \in$ $\stackrel{H}{m}_{m}\left(S_{n}\left(x^{0} ; \epsilon\right)\right) \subset H_{m}(\Omega) \subset V$, where $S_{n}\left(x^{0} ; \epsilon\right)=\left\{x \in R^{n}|| x_{i}-x_{i}^{0} \mid<\epsilon^{1 / m i}\right.$, $1 \leqslant i \leqslant n$. Then it is readily verified that

$$
\begin{aligned}
a(v, v)-\lambda(v, v) & =a(v, v)-a\left(u, \eta_{\epsilon} v\right)+a\left(u, \eta_{\epsilon} v\right)-\lambda\left(\eta_{\epsilon} u, v\right) \\
& =a(v, v)-a\left(u, \eta_{\epsilon} v\right) .
\end{aligned}
$$

Recalling the law of sine and noting that $a(v, v) \geqslant 0$ and that

$$
\frac{d(\lambda)}{|\lambda|}= \begin{cases}\sin (\arg \lambda), & \text { if } \operatorname{Re} \lambda>0, \\ 1, & \text { if } \operatorname{Re} \lambda \leqslant 0,\end{cases}
$$

we have, for some constant $C$,

$$
|a(v, v)-\lambda(v, v)| \geqslant \max \left\{\delta\|v\|_{m}^{2},|\lambda|\|v\|_{0}^{2}\right\} d(\lambda) /|\lambda| \geqslant C B(v)^{2} d(\lambda) /|\lambda|,
$$

where $B(v)=\|v\|_{m}+|\lambda|^{1 / 2}\|v\|_{0}$.

From (3.1) and (3.2) it follows that 


$$
\begin{aligned}
& C B(v)^{2} d(\lambda) /|\lambda| \leqslant\left|a(v, v)-a\left(u, \eta_{\epsilon} v\right)\right| \\
& \quad=\left|\int_{\Omega} \sum_{(1,1)} a_{\alpha \beta}(x)\left(D^{\alpha}\left(\eta_{\epsilon} u\right) \overline{D^{\beta} v}-D^{\alpha} u \overline{D^{\beta}\left(\eta_{\epsilon} v\right)}\right) d x\right| \\
& \quad \leqslant\left|\sum_{(1,1)}^{\prime}\right|+\left|\sum_{|\alpha: m| \leqslant 1-a,|\beta: m|=1}\right|+\left|\sum_{|\alpha: m|=1,|\beta: m| \leqslant 1-a}\right|+\left|\sum_{(1-a, 1-a)}\right| \\
& =I_{1}+I_{2}+I_{3}+I_{4} .
\end{aligned}
$$

We now carry out $I_{1}$ as follows:

$$
\begin{aligned}
& \mathrm{I}_{1} \leqslant\left|\sum_{(1,1)}^{\prime} \int_{\Omega} a_{\alpha \beta}(x)\left(\eta_{\epsilon} D^{\alpha} u \overline{D^{\beta} v}-D^{\alpha} u\left(\eta_{\epsilon} \overline{D^{\beta} v}\right)\right) d x\right| \\
&+\mid \sum_{(1,1)}^{\prime} \int_{\Omega} a_{\alpha \beta}(x)\left(\sum_{1-2 b<|\gamma: m| \leqslant 1-b}\left(\begin{array}{c}
\alpha \\
\gamma
\end{array}\right) D^{\alpha-\gamma} \eta_{\epsilon} D^{\gamma} u \overline{D^{\beta} v}\right. \\
&\left.-D^{\alpha} u \sum_{1-2 b<|\delta: m| \leqslant 1-b}\left(\begin{array}{c}
\beta \\
\delta
\end{array}\right) D^{\beta-\delta} \eta_{\epsilon} \overline{D^{\delta} v}\right) d x \mid \\
&+\mid \sum_{(1,1)}^{\prime} \int_{\Omega} a_{\alpha \beta}(x)\left(\sum_{|\gamma: m| \leqslant 1-2 b}\left(\begin{array}{l}
\alpha \\
\gamma
\end{array}\right) D^{\alpha-\gamma} \eta_{\epsilon} D^{\gamma} u \overline{D^{\beta} v}\right. \\
&\left.-D^{\alpha} u \sum_{|\delta: m| \leqslant 1-2 b}\left(\begin{array}{c}
\beta \\
\delta
\end{array}\right) D^{\beta-\delta} \eta_{\epsilon} \overline{D^{\delta} v}\right) d x \mid \\
&=\mathrm{I}_{11}+\mathrm{I}_{12}+\mathrm{I}_{13} .
\end{aligned}
$$

Clearly $\mathrm{I}_{11}=0$. Since $\operatorname{Sup}_{x \in \Omega}\left|D^{\alpha} \eta_{\epsilon}(x)\right| \leqslant C \epsilon^{-|\alpha: m|}$,

$$
\mathrm{I}_{12} \leqslant C\left(\sum_{1-2 b<s \leqslant 1-b} \epsilon^{-(1-s)}\|u\|_{s m}\|v\|_{m}+\sum_{1-2 b \leqslant t \leqslant 1-b} \epsilon^{-(1-t)}\|u\|_{m}\|v\|_{t m}\right),
$$

and, because $\|r f\|_{-m} \leqslant\|f\|_{V^{*}}$, it follows from Lemmas 2.6 and 2.7 that for $f \in V^{*}$

$$
\begin{aligned}
\mathrm{I}_{12} \leqslant C\left(\sum_{s} \epsilon^{-(1-s)}|\lambda|^{1 / 2(1+s)} d(\lambda)^{-1}\|f\|_{V^{*}}\|v\|_{m}\right. \\
\left.+\sum_{t} \epsilon^{-(1-t)}|\lambda|^{1 / 2(1+t)} d(\lambda)^{-1} B(v)\|f\|_{V^{*}}\right) .
\end{aligned}
$$

(Note that $\|u\|_{m} \leqslant 2\left\|(A-\lambda)^{-1} f\right\|_{m} \leqslant C|\lambda| d(\lambda)^{-1}\|f\|_{V^{*}}$ ) Substituting $s=t=1-b$ in the above and noting that $\|v\|_{m} \leqslant B(v)$, we have

$$
\mathrm{I}_{12} \leqslant C \epsilon^{-b}|\lambda|^{1-b / 2} d(\lambda)^{-1}\|f\|_{V^{*}} B(v) .
$$

Similarly, for $f \in L^{2}(\Omega)$ we have

$$
\mathrm{I}_{12} \leqslant C \epsilon^{-b}|\lambda|^{1 / 2-b / 2} d(\lambda)^{-1}\|f\|_{0} B(v) .
$$

Similar to the bounds for $I_{12}$ we find 


$$
\mathrm{I}_{13} \leqslant \begin{cases}C \epsilon^{-2 b}|\lambda|^{1-b} d(\lambda)^{-1}\|f\|_{V^{*}} B(v), & \text { for } f \in V^{*}, \\ C \epsilon^{-2 b}|\lambda|^{1 / 2-b} d(\lambda)^{-1}\|f\|_{0} B(v), & \text { for } f \in L^{2}(\Omega) .\end{cases}
$$

Since $\epsilon^{-1}|\lambda|^{-1 / 2} \leqslant 1$ implies $\epsilon|\lambda|^{1 / 2} \geqslant 1,\left(\epsilon|\lambda|^{1 / 2}\right)^{-2 b} \leqslant\left(\epsilon|\lambda|^{1 / 2}\right)^{-b}$. Thus $I_{13} \leqslant I_{12}$. Consequently, we get

$$
\mathrm{I}_{1} \leqslant C \epsilon^{-b}|\lambda|^{1-b / 2} d(\lambda)^{-1}\|f\|_{V^{*} B(v),} \text { for } f \in V^{*} \text {, }
$$

and

$$
I_{1} \leqslant C \epsilon^{-b}|\lambda|^{\frac{1}{2}-b / 2} d(\lambda)^{-1}\|f\|_{0} B(v), \text { for } f \in L^{2}(\Omega) .
$$

For $I_{2}$ we have

$$
\begin{aligned}
\mathrm{I}_{2}= & \underset{\substack{|\alpha: m|<1-a,|\beta: m|=1}}{ } \int_{\Omega} a_{\alpha \beta}(x)\left(\sum_{\gamma<\alpha}\left(\begin{array}{c}
\alpha \\
\gamma
\end{array}\right) D^{\alpha-\gamma} \eta_{\epsilon} D^{\gamma} u \overline{D^{\beta} v}\right. \\
& \left.-D^{\alpha} u \sum_{\delta<\beta}\left(\begin{array}{l}
\beta \\
\delta
\end{array}\right) D^{\beta-\delta} \eta_{\epsilon} \overline{D^{\delta} v}\right) d x \mid .
\end{aligned}
$$

(Note that the term for $\gamma=\alpha$ and $\delta=\beta$ is zero.)

Since $|\alpha-\gamma: m|=|\alpha: m|-|\gamma: m| \leqslant 1-a-s$ for $\gamma<\alpha$ and $|\beta-\delta: m|$ $=|\beta: m|-|\delta: m| \leqslant 1-t$ for $\delta<\beta$ imply that $s \leqslant 1-(a+b)$ and $t \leqslant 1-b$ respectively, it follows from Lemmas 2.6 and 2.7 that for $f \in V^{*}$

$$
\begin{aligned}
\mathrm{I}_{2} \leqslant C\left(\sum_{s \leqslant 1-(a+b)} \epsilon^{-(1-a-s)}\|u\|_{s m}\|v\|_{m}\right. \\
\left.\quad+\sum_{t<1-b} \epsilon^{-(1-t)}\|u\|_{(1-a) m}\|v\|_{t m}\right) \\
\leqslant C \epsilon^{-b}|\lambda|^{1-(a+b) / 2} d(\lambda)^{-1}\|f\|_{V^{*} B(v) .}
\end{aligned}
$$

Similarly, we find

$$
\mathrm{I}_{2} \leqslant C \epsilon^{-b}|\lambda|^{1 / 2-(a+b) / 2} d(\lambda)^{-1}\|f\|_{0} B(v), \text { for } f \in L^{2}(\Omega) .
$$

It is evident that the bound of $I_{3}$ is the same as that of $I_{2}$. For $I_{4}$ we have

$$
\begin{aligned}
\mathrm{I}_{4} \leqslant C\left(\sum_{s \leqslant 1-(a+b)} \epsilon^{-(1-a-s)}\|u\|_{s m}\|v\|_{(1-a) m}\right. \\
\left.\quad+\sum_{t \leqslant 1-(a+b)} \epsilon^{-(1-a-t)}\|u\|_{(1-a) m}\|v\|_{t m}\right) \\
\leqslant C \epsilon^{-b}|\lambda|^{1-(a+b) / 2}|\lambda|^{-a / 2} d(\lambda)^{-1}\|f\|_{V^{*}} B(v), \text { for } f \in V^{*} .
\end{aligned}
$$

And again

$$
\mathrm{I}_{4} \leqslant C \epsilon^{-b}|\lambda|^{1 / 2-(a+b) / 2}|\lambda|^{-a / 2} d(\lambda)^{-1}\|f\|_{0} B(v), \text { for } f \in L^{2}(\Omega) .
$$

If $|\lambda| \geqslant 1,|\lambda|^{-a / 2} \leqslant 1$ and

$$
\epsilon^{-b}|\lambda|^{1-(a+b) / 2}|\lambda|^{-a / 2} \leqslant \epsilon^{-b}|\lambda|^{1-(a+b) / 2} \leqslant \epsilon^{-b}|\lambda|^{1-b / 2} ;
$$

thus, from (3.5), (3.6) and (3.7), we get 


$$
\begin{aligned}
& \mathrm{I}_{1}+\mathrm{I}_{2}+\mathrm{I}_{3}+\mathrm{I}_{4} \\
& \leqslant C\left(\epsilon^{-b}|\lambda|^{1-b / 2}+2 \epsilon^{-b}|\lambda|^{1-(a+b) / 2}+\epsilon^{-b}|\lambda|^{1-(a+b) / 2}|\lambda|^{-a / 2}\right) \\
& \cdot d(\lambda)^{-1}\|f\|_{V^{*}} B(v) \\
& \leqslant C \epsilon^{-b}|\lambda|^{1-b / 2} d(\lambda)^{-1}\|f\|_{V^{*}} B(v),
\end{aligned}
$$

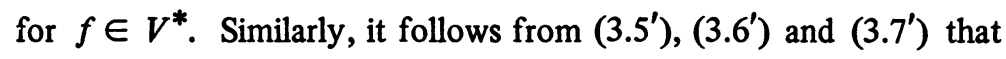

$$
\mathrm{I}_{1}+\mathrm{I}_{2}+\mathrm{I}_{3}+\mathrm{I}_{4} \leqslant C \epsilon^{-b}|\lambda|^{1 / 2-b / 2} d(\lambda)^{-1}\|f\|_{0} B(v),
$$

for $f \in L^{2}(\Omega)$. From (3.3), (3.8) and (3.8') we have

$$
B(v)^{2} \frac{d(\lambda)}{|\lambda|} \leqslant\left\{\begin{array}{l}
C \epsilon^{-b}|\lambda|^{1-b / 2} d(\lambda)^{-1}\|f\|_{V^{*}} B(v), \\
C \epsilon^{-b}|\lambda|^{1 / 2-b / 2} d(\lambda)^{-1}\|f\|_{0} B(v),
\end{array}\right.
$$

which implies that

$$
B(v) \leqslant\left\{\begin{array}{l}
C F(\epsilon, \lambda, 1)|\lambda| d(\lambda)^{-1}\|f\|_{V^{*}} \\
C F(\epsilon, \lambda, 1)|\lambda|^{1 / 2} d(\lambda)^{-1}\|f\|_{0} .
\end{array}\right.
$$

Consequently, we obtain

$$
\begin{aligned}
& \|v\|_{m} \leqslant K_{1} F(\epsilon, \lambda, 1)|\lambda| d(\lambda)^{-1}\|f\|_{V^{*}}, \\
& \|v\|_{0} \leqslant K_{1} F(\epsilon, \lambda, 1)|\lambda|^{1 / 2} d(\lambda)^{-1}\|f\|_{V^{*}}, \\
& \|v\|_{m} \leqslant K_{1} F(\epsilon, \lambda, 1)|\lambda|^{1 / 2} d(\lambda)^{-1}\|f\|_{0}, \\
& \|v\|_{0} \leqslant K_{1} F(\epsilon, \lambda, 1) d(\lambda)^{-1}\|f\|_{0} .
\end{aligned}
$$

Hence the lemma is proved for $j=1$. We proceed inductively. Assuming now that the lemma has been proved for $j=k$, we then pick another function $\zeta \in \Lambda$ such that $\zeta(x)=1$ for any $x \in \operatorname{supp}(\eta)$ and write $\zeta_{\epsilon}(x)=$ $\zeta\left(\left(x-x^{0}\right) / \epsilon^{(1 / m)}\right)$. Letting $\zeta_{\epsilon} u=(A-\lambda)^{-1} f-\left(A_{0}-\lambda\right)^{-1}(r f)$ and $v=$ $\eta_{\epsilon} \zeta_{\epsilon} u=S_{\lambda_{\epsilon}} f$, we have (similar to (3.1), (3.2), (3.3))

$$
\begin{aligned}
& C B(v)^{2} \frac{d(\lambda)}{|\lambda|} \leqslant\left|a(v, v)-a\left(\zeta_{\epsilon} u, \eta_{\epsilon} v\right)\right| \\
& =\mid \int_{\Omega} \sum_{(1,1)} a_{\alpha \beta}(x) \sum_{\gamma<\alpha}\left(\begin{array}{l}
\alpha \\
\gamma
\end{array}\right) D^{\alpha-\gamma} \eta_{\epsilon} D^{\gamma}\left(\zeta_{\epsilon} u\right) \overline{D^{\beta} v} \\
& \left.\quad-D^{\alpha}\left(\zeta_{\epsilon} u\right) \sum_{\delta<\beta}\left(\begin{array}{c}
\beta \\
\delta
\end{array}\right) D^{\beta-\delta} \eta_{\epsilon} \overline{D^{\delta} v}\right) d x \mid \\
& \leqslant\left|\sum_{(1,1)}^{\prime}\right|+\left|\sum_{\substack{|\alpha: m|<1-a,|\beta: m|=1}}\right|+\left|\sum_{\substack{|\alpha: m|=1,|\beta: m| \leqslant 1-a}}\right|+\left|\sum_{(1-a, 1-a)}\right| \\
& =\mathrm{I}_{1}^{\prime}+\mathrm{I}_{2}^{\prime}+\mathrm{I}_{3}^{\prime}+\mathrm{I}_{4}^{\prime} .
\end{aligned}
$$


As in the proof of the case $j=1$, we find that

$$
\begin{aligned}
\mathrm{I}_{1}^{\prime} & \leqslant C \epsilon^{-b}|\lambda|^{-b / 2} B(v) B\left(\zeta_{\epsilon} u\right), \\
\mathrm{I}_{2}^{\prime}, \mathrm{I}_{3}^{\prime} & \leqslant C \epsilon^{-b}|\lambda|^{-(a+b) / 2} B(v) B\left(\zeta_{\epsilon} u\right), \\
\mathrm{I}_{4}^{\prime} & \leqslant C \epsilon^{-b}|\lambda|^{-(a+b) / 2}|\lambda|^{-a / 2} B(v) B\left(\zeta_{\epsilon} u\right) .
\end{aligned}
$$

In the same way as for the case $j=1$, we conclude from the above results that

$$
B(v)^{2} \frac{d(\lambda)}{|\lambda|} \leqslant C \epsilon^{-b}|\lambda|^{-b / 2} B(v) B\left(\zeta_{\epsilon} u\right) .
$$

But, from the induction hypothesis with $\zeta$ in place of $\eta$ it follows that

$$
B\left(\zeta_{\epsilon} u\right) \leqslant\left\{\begin{array}{l}
K_{k} F(\epsilon, \lambda, k)|\lambda| d(\lambda)^{-1}\|f\|_{V^{*}}, \\
K_{k} F(\epsilon, \lambda, k)|\lambda|^{1 / 2} d(\lambda)^{-1}\|f\|_{0} .
\end{array}\right.
$$

From (3.9) and (3.10) it follows that

$$
B(v) \frac{d(\lambda)}{|\lambda|} \leqslant\left\{\begin{array}{l}
C \epsilon^{-b}|\lambda|^{-b / 2} K_{k} F(\epsilon, \lambda, k)|\lambda| d(\lambda)^{-1}\|f\|_{V^{*}}, \\
C \epsilon^{-b}|\lambda|^{-b / 2} K_{k} F(\epsilon, \lambda, k)|\lambda|^{1 / 2} d(\lambda)^{-1}\|f\|_{0},
\end{array}\right.
$$

which implies that

$$
B(v) \leqslant\left\{\begin{array}{l}
K_{k+1} F(\epsilon, \lambda, k+1)|\lambda| d(\lambda)^{-1}\|f\|_{V^{*}}, \\
K_{k+1} F(\epsilon, \lambda, k+1)|\lambda|^{1 / 2} d(\lambda)^{-1}\|f\|_{0},
\end{array}\right.
$$

where $K_{k+1}=C K_{k}$. The completion of the induction step follows immediately from (3.11), and the lemma is proved.

As in Lemma 2.4 let $\Omega$ be an $n$-box or $R^{n}$. Set $c<1$. Moreover, let $M_{\lambda_{\epsilon}}(x, y), K_{\lambda}(x, y)$ and $K_{\lambda}^{0}(x, y)$ be the kernels of the operators $S_{\lambda_{\epsilon}}$, $(A-\lambda)^{-1}$ and $\left(A_{0}-\lambda\right)^{-1}$ respectively. Then clearly we have the relation

$$
M_{\lambda_{\epsilon}}(x, y)=\eta_{\epsilon}(x)\left\{K_{\lambda}(x, y)-K_{\lambda}^{0}(x, y)\right\} .
$$

A lemma which is similar to Lemma 4.2 given on p. 332 of [9] is now stated below.

LEMMA 3.2. Corresponding to any $p>0$ there exists a constant $C=C(p)$ so that for any $\lambda$ with $|\lambda| \geqslant 1$ and for any $x^{0}$ in $\Omega$ the following inequality holds:

$$
\left|K_{\lambda}\left(x^{0}, x^{0}\right)-K_{\lambda}^{0}\left(x^{0}, x^{0}\right)\right| \leqslant C \frac{|\lambda|^{c}}{d(\lambda)}\left(\frac{|\lambda|^{1-b / 2}}{\delta\left(x^{0}\right) d(\lambda)}\right)^{p},
$$

where $c<1$ and $\delta\left(x^{0}\right)=\min \left\{1\right.$, dist $\left.\left(x^{0}, \partial \Omega\right)\right\}$.

4. Approximation of coefficients by smooth functions. We shall approximate the coefficients $a_{\alpha \beta}$ by functions in $C^{\infty}\left(R^{n}\right)$ so that we can apply Kannai's results of [8]. Let $\widetilde{\rho}$ denote the real-valued even function in $C_{0}^{\infty}\left(R^{1}\right)$ 
for which $\operatorname{supp}(\widetilde{\rho})$ is contained in the set $\left\{x_{i} \in R^{1}|| x_{i} \mid \leqslant 1 / \sqrt{n}\right\}, 1 \leqslant i \leqslant n$.

We write for $x=\left(x_{1}, \cdots, x_{n}\right) \in R^{n}$

$$
\rho_{\epsilon}(x)=\epsilon^{-c / 2} \prod_{j=1}^{n} \widetilde{\rho}\left(\frac{x_{j}}{\epsilon^{1 / m_{j}}}\right) .
$$

Set $\int_{R^{n}} \rho_{\epsilon}(x) d x=C$. (We may consider $C=1$ for convenience of calculation.)

We now state, without proofs, Lemmas 4.1-4.4, which are similar to Lemmas 5.1-5.4 given on pp. 333-336 of [9].

Lemma 4.1. Let $\Omega$ be an $n$-box or $R^{n}$. For $f \in C^{2}(\Omega), x^{0} \in \Omega$, and for any positive numbers $\epsilon, \delta<1$, we set

$$
f_{0}(x)= \begin{cases}\sum_{|\alpha| \leqslant 2} \frac{1}{\alpha !}\left(x-x^{0}\right)^{\alpha} \partial_{x}^{\alpha} f\left(x^{0}\right), & \text { if }\left|x_{i}-x_{i}^{0}\right| \leqslant \delta^{1 / m_{i}}, \\ \sum_{|\alpha| \leqslant 2} \frac{1}{\alpha !}\left(x^{1}-x^{0}\right)^{\alpha} \partial_{x}^{\alpha} f\left(x^{0}\right), & \text { if }\left|x_{i}-x_{i}^{0}\right|>\delta^{1 / m_{i}},\end{cases}
$$

where $x^{1}$ is the point of intersection of the right parallelepiped $\left\{x \in R^{n} \mid\right.$ $\left.\left|x_{i}-x_{i}^{0}\right|=\delta^{1 / m_{i}}, 1 \leqslant i \leqslant n\right\}$ with the line segment connecting $x^{0}$ and $x$. Then

(i) $\rho_{\epsilon} * f_{0}$ is a function in $C^{\infty}\left(R^{n}\right)$;

(ii) when $\epsilon<\delta$, we have $\rho_{\epsilon} * f_{0}(x)=f_{0}(x)+C_{\epsilon}\left(x^{0}\right)$ in the set $\left\{x \in R^{n}|| x_{i}-x_{i}^{0} \mid<\delta^{1 / m_{i}}-\epsilon^{1 / m_{i}}, 1 \leqslant i \leqslant n\right\}$, where $C_{\epsilon}\left(x^{0}\right)$ is independent of $x$ :

$$
C_{\epsilon}\left(x^{0}\right)=\sum_{|\alpha|=2} \frac{1}{\alpha !} \partial^{\alpha} f\left(x^{0}\right) \int_{R^{n}} z^{\alpha} \rho_{\epsilon}(z) d z
$$

and satisfies

$$
\left|C_{\epsilon}\left(x^{0}\right)\right| \leqslant \epsilon^{2 b} \sum_{|\alpha|=2} \frac{1}{\alpha !}\left|\partial_{x}^{\alpha} f\left(x^{0}\right)\right| ;
$$

(iii) for any $x \in R^{n}$ we have

$$
\left|\rho_{\epsilon} * f_{0}(x)-f\left(x^{0}\right)\right| \leqslant 2 \delta^{b} \sum_{|\alpha|=1}\left|\partial_{x}^{\alpha} f\left(x^{0}\right)\right|+2 \delta^{2 b} \sum_{|\alpha|=2}\left|\partial_{x}^{\alpha} f\left(x^{0}\right)\right| .
$$

Lemma 4.2. Let $\Omega, x^{0}, x^{1}, \epsilon, \delta$ be the same as in Lemma 4.1. For $f \in C^{1}(\Omega)$ we set

$$
f_{0}(x)= \begin{cases}\sum_{|\alpha|=1}\left(x-x^{0}\right)^{\alpha} \partial_{x}^{\alpha} f\left(x^{0}\right), & \text { for }\left|x_{i}-x_{i}^{0}\right| \leqslant \delta^{1 / m_{i}}, \\ \sum_{|\alpha|=1}\left(x^{1}-x^{0}\right)^{\alpha} \partial_{x}^{\alpha} f\left(x^{0}\right), & \text { for }\left|x_{i}-x_{i}^{0}\right|>\delta^{1 / m_{i}} .\end{cases}
$$

Then

(i) $\rho_{\epsilon} * f_{0}$ is a function in $C^{\infty}\left(R^{n}\right)$; 


$$
\begin{aligned}
& \text { (ii) when } \epsilon<\delta, \rho_{\epsilon} * f_{0}(x)=f_{0}(x) \text { in the set }\left\{x \in R^{n}|| x_{i}-x_{i}^{0} \mid<\right. \\
& \left.\delta^{1 / m_{i}}-\epsilon^{1 / m_{i}}, 1 \leqslant i \leqslant n\right\} ;
\end{aligned}
$$

(iii) for any $x \in R^{n}$ we have

$$
\left|\rho_{\epsilon} * f_{0}(x)-f_{0}\left(x^{0}\right)\right| \leqslant 2 \delta^{b} \sum_{|\alpha|=1}\left|\partial_{x}^{\alpha} f\left(x^{0}\right)\right| .
$$

We would like to modify the coefficients of $a(u, v)$, but leave semiellipticity of $a(u, v)$ invariant.

Suppose that the coefficients of $a(u, v)$ satisfy the smoothness condition s-(4). Select $\epsilon^{\prime}$ and $\delta$ so that $0<\epsilon^{\prime}<\delta<1$, then pick any fixed point $x^{0}$ of $\Omega$. We shall apply Lemma 4.1 to $a_{\alpha \beta}$ for $|\alpha+\beta: m|=2$ and Lemma 4.2 to $a_{\alpha \beta}$ for $|\alpha+\beta: m|=2-a$. For $|\alpha+\beta: m|=2$, let $a_{\alpha \beta}^{0}$ and $C_{\epsilon^{\prime}}^{\alpha \beta}\left(x^{0}\right)$ be the function and the constant defined by (4.1) and (4.2) respectively with $f$ and $\epsilon$ replaced by $a_{\alpha \beta}$ and $\epsilon^{\prime}$, and set $a_{\alpha \beta}^{1}\left(x^{0}\right)=\rho_{\epsilon^{\prime}} * a_{\alpha \beta}^{0}\left(x^{0}\right)-C_{\epsilon^{\prime}}^{\alpha \beta}\left(x^{0}\right)$. For $|\alpha+\beta: m|=2-a$, let $a_{\alpha \beta}^{0}$ be the function defined by (4.3) with $f$ replaced by $a_{\alpha \beta}$ and set $a_{\alpha \beta}^{1}\left(x^{0}\right)=\rho_{\epsilon^{\prime}} * a_{\alpha \beta}^{0}\left(x^{0}\right)$. For $|\alpha+\beta: m|=2-2 a$ and $|\alpha+\beta: m|=2-3 a$, we put $a_{\alpha \beta}^{1}(x)=a_{\alpha \beta}\left(x^{0}\right)$ and $a_{\alpha \beta}^{1}(x)=0$ respectively. We shall consider the following symmetric sesquilinear form

$$
a_{1}(u, v)=\sum_{(1,1)} \int_{\Omega} a_{\alpha \beta}^{1}(x) D^{\alpha} u \overline{D^{\beta} v} d x .
$$

LEMMA 4.3. Let $\Omega$ be an $n$-box or $R^{n}$. Then there exist two positive constants $C_{0}^{\prime}$ and $C$ such that

$$
a_{1}(u, u) \geqslant C_{0}^{\prime}\|u\|_{m}^{2}-C\|u\|_{0}^{2}
$$

for any $u \in \dot{H}_{m}(\Omega)$ provided that, as stated above, $\delta$ and $\epsilon^{\prime}$ are sufficiently small and independent of $x^{0}$ (an arbitrary point in $\Omega$ ) with $0<\epsilon^{\prime}<\delta<1$.

Next, consider the case when the coefficients $a_{\alpha \beta}$ satisfy the condition $s$-(3). For $|\alpha+\beta: m|=2$, letting $a_{\alpha \beta}^{0}$ be defined by (4.3) with $a_{\alpha \beta}$ in place of $f$, we put $a_{\alpha \beta}^{2}(x)=\rho_{\epsilon^{\prime}} * a_{\alpha \beta}^{0}(x)$. For $|\alpha+\beta: m|=2-a$ and $|\alpha+\beta: m|=$ $2-2 a$, we put $a_{\alpha \beta}^{2}(x)=a_{\alpha \beta}\left(x^{0}\right)$ and $a_{\alpha \beta}^{2}(x)=0$ respectively. After the coefficients $a_{\alpha \beta}^{2}$ are so defined we construct the sesquilinear form as follows:

$$
a_{2}(u, v)=\sum_{(1,1)} \int_{\Omega} a_{\alpha \beta}^{2}(x) D^{\alpha} u \overline{D^{\beta} v} d x
$$

for any $u, v \in \dot{H}_{m}(\Omega)$. In the case when the coefficients $a_{\alpha \beta}$ satisfy the condition s-(2), we put $a_{\alpha \beta}^{3}(x)=a_{\alpha \beta}\left(x^{0}\right)$ for $|\alpha+\beta: m|=2$. Then let $a_{3}(u, v)$ be the sesquilinear form on $H_{m}(\Omega) \times H_{m}(\Omega)$ defined by

for any $u, v \in \dot{H}_{m}(\Omega)$.

$$
a_{3}(u, v)=\sum_{(1,1)}^{\prime} \int_{\Omega} a_{\alpha \beta}(x) D^{\alpha} u \overline{D^{\beta} v} d x
$$

Lemma 4.4. Let $\Omega$ be an $n$-box or $R^{n}$. Then there exist two positive 
constants $C_{0}^{\prime}$ and $C$ such that for $i=2,3$

$$
a_{i}(u, u) \geqslant C_{0}^{\prime}\|u\|_{m}^{2}-C\|u\|_{0}^{2}
$$

for any $u \in \stackrel{\circ}{H}_{m}(\Omega)$, provided that $\delta$ and $\epsilon^{\prime}$ are sufficiently small and independent of $x^{0}$ with $0<\epsilon^{\prime}<\delta<1$.

With a minor change of notations a method given on pp. 87-89 of [1] can be readily adopted to prove the following lemma.

LEMMA 4.5. Let $a_{\alpha \beta}$ be bounded and measurable in any open region $\Omega$ for $|\alpha: m| \leqslant 1,|\beta: m| \leqslant 1$. Let $C_{0}>0$ and $C^{\prime} \geqslant 0$ be given constants. If for all $\phi \in C_{0}^{\infty}(\Omega)$

$$
\operatorname{Re} a(\phi, \phi) \geqslant C_{0}\|\phi\|_{m}^{2}-C^{\prime}\|\phi\|_{0}^{2},
$$

then for almost all $x$ in $\Omega$ and for any real vector $\xi$,

$$
\operatorname{Re} \sum_{(1,1)}^{\prime} a_{\alpha \beta}(x) \xi^{\alpha+\beta} \geqslant C_{0} \mu(\xi) \text {, where } \mu(\xi)=\sum_{j=1}^{n}\left|\xi_{j}\right|^{2 m_{j}} \text {. }
$$

5. Effect of smoothing coefficients of the kernels on $\stackrel{\circ}{H}_{m}(\Omega)$. Suppose that $\epsilon^{\prime}$ and $\delta$ are given sufficiently small with $0<\epsilon^{\prime}<\delta<1$, as above. In the previous section the semielliptic sesquilinear forms $a_{i}(u, v)$ have been defined by means of the smooth coefficients $a_{\alpha \beta}^{i}, i=1,2,3$. According to the Lax-Milgram theorem, there exist operators $A_{i}$ associated with the forms $a_{i}(u, v)$ restricted to $\stackrel{\circ}{H}_{m}(\Omega) \times \dot{H}_{m}(\Omega)$, that is, $a_{i}(u, v)=\left(A_{i} u, v\right)$ for any $u, v \in \stackrel{\circ}{H}_{m}(\Omega), i=1,2,3$. Consequently, for sufficiently large $|\lambda|$ the operators

$$
A_{i}-\lambda: \stackrel{\circ}{H}_{m}(\Omega) \rightarrow H_{-m}(\Omega)
$$

defined by

$$
a_{i}(u, v)-\lambda(u, v)=\left(\left(A_{i}-\lambda\right) u, v\right)
$$

for $u, v \in \dot{H}_{m}(\Omega)$, possess bounded inverses $\left(A_{i}-\lambda\right)^{-1}$ respectively, $i=1,2,3$. In order to estimate the difference between the resolvent kernels of $A_{0}$ and those of $A_{1}$, we set

$$
S_{\lambda_{\epsilon}}^{1} f=\eta_{\epsilon}\left\{\left(A_{0}-\lambda\right)^{-1}-\left(A_{1}-\lambda\right)^{-1}\right\} f
$$

for $f \in H_{-m}(\Omega)$, where $\epsilon$ is an arbitrary positive number and $\eta_{\epsilon}(x)=$ $\eta\left(\left(x-x^{0}\right) / \epsilon^{(1 / m)}\right)$ for $\eta \in \Lambda$ as before. For an operator $S$ on $H_{-m}(\Omega)$ to $\stackrel{\circ}{m}_{m}(\Omega)$ we denote by

$$
\|S\|_{(-m, m)}, \quad\|S\|_{(-m, 0)}, \quad\|S\|_{(0, m)} \text { and }\|S\|_{(0,0)}
$$

the norms of $S$ considered as an operator on $H_{-m}(\Omega)$ to $\stackrel{\circ}{m}_{m}(\Omega)$, on $H_{-m}(\Omega)$ to $L^{2}(\Omega)$, to $\stackrel{\circ}{H}_{m}(\Omega)$ and on $L^{2}(\Omega)$ to $L^{2}(\Omega)$ respectively. 
LEMMA 5.1. If $\epsilon^{-b}|\lambda|^{1-b / 2} d(\lambda)^{-1} \leqslant 1$ with $0<\epsilon<1,|\lambda| \geqslant 1$, then for any positive integer $j$ there exists a positive constant $K_{j}$, which is independent of $x^{0}, \epsilon$ and $\lambda$, such that

where

$$
\begin{aligned}
\left\|S_{\lambda_{\epsilon}}^{1}\right\|_{(-m, m)} & \leqslant K_{j} R_{1}(\epsilon, \lambda, j), \\
\left\|S_{\lambda_{\epsilon}}^{1}\right\|_{(-m, 0)},\left\|S_{\lambda_{\epsilon}}^{1}\right\|_{(0, m)} & \leqslant K_{j} R_{1}(\epsilon, \lambda, j)|\lambda|^{-1 / 2}, \\
\left\|S_{\lambda_{\epsilon}}^{1}\right\|_{(0,0)} & \leqslant K_{j} R_{1}(\epsilon, \lambda, j)|\lambda|^{-1}
\end{aligned}
$$

$$
R_{1}(\dot{\epsilon}, \lambda, j)=\epsilon^{2 b+h b}\left(1+\epsilon^{-h b}|\lambda|^{-a / 2}\right)\left(\frac{|\lambda|}{d(\lambda)}\right)^{2}+\left(\frac{|\lambda|^{1-b / 2}}{\epsilon^{b} d(\lambda)}\right)^{j} \frac{|\lambda|}{d(\lambda)} .
$$

Since the proof of the lemma is similar to that of Lemma 6.1 given on pp. 336-338 of [9], we merely state the changes of bounds of $\mid a_{\alpha \beta}(x)-$ $a_{\alpha \beta}^{1}(x) \mid$ which are necessary in the semielliptic case. First consider $a_{\alpha \beta}$ for $|\alpha+\beta: m|=2$. Let $\epsilon$ be such that $0<\epsilon \leqslant \min \left\{\epsilon_{0}, \delta-\epsilon^{\prime}\right\}$ where $\epsilon_{0}=$ $\operatorname{dist}\left(\Omega, \partial \Omega_{1}\right)$. Then in view of Lemma 4.1, we get, for $\left|x_{i}-x_{i}^{0}\right|<\epsilon^{1 / m_{i}}$, $1 \leqslant i \leqslant n$,

$$
a_{\alpha \beta}^{1}(x)=a_{\alpha \beta}^{0}(x)=\sum_{|\lambda| \leqslant 2} \frac{1}{\gamma !}\left(x-x^{0}\right)^{\gamma} \partial_{x}^{\gamma} a_{\alpha \beta}\left(x^{0}\right) .
$$

Hence it follows from the Taylor expansion of $a_{\alpha \beta}$ at $x=x^{0}$ that

$$
\left|a_{\alpha \beta}(x)-a_{\alpha \beta}^{1}(x)\right| \leqslant C \epsilon^{2 b+h b} .
$$

Replacing $C$ by another constant if necessary we find that (5.1) is true without any restriction on $\epsilon>0$. Similarly, for $|\alpha+\beta: m|=2-a$ and $|\alpha+\beta: m|=$ $2-2 a$, we have

$$
\left|a_{\alpha \beta}(x)-a_{\alpha \beta}^{1}(x)\right| \leqslant C \epsilon^{b+h b} \text { and }\left|a_{\alpha \beta}(x)-a_{\alpha \beta}^{1}(x)\right| \leqslant C \epsilon^{h b}
$$

respectively, if $\left|x_{i}-x_{i}^{0}\right|<\epsilon^{1 / m_{i}}, 1 \leqslant i \leqslant n$.

Now the proof of the lemma follows immediately from similar arguments of [9].

Let $A_{i}$ be the operator associated with the sesquilinear form $a_{i}(u, v)$ and denote by $K_{\lambda}^{i}(x, y)$ the resolvent kernel of $A_{i}, i=1,2,3$.

Lemma 5.2. Let $\Omega$ be an $n$-box or $R^{n}$. Set $c<1$. For any positive integer $j$, there exists a positive constant $C=C(j)$ so that, for any selected $x^{0} \in \Omega, \epsilon$ and $\lambda$ for which $0<\epsilon<1,|\lambda| \geqslant 1$ and $\epsilon^{-b}|\lambda|^{1-b / 2} d(\lambda)^{-1} \leqslant 1$, it is the case that

$$
\left|K_{\lambda}^{0}\left(x^{0}, x^{0}\right)-K^{i}\left(x^{0}, x^{0}\right)\right|<C|\lambda|^{c-1} R_{i}(\epsilon, \lambda, j) \text { under s-(5-i), }
$$




$$
\begin{array}{r}
R_{i}(\epsilon, \lambda, j)=\epsilon^{2 b+h b-(i-1)}\left(1+\epsilon^{-h b}|\lambda|^{-a / 2}\left(\frac{|\lambda|}{d(\lambda)}\right)^{2}+\left(\frac{|\lambda|^{1-b / 2}}{\epsilon^{b} d(\lambda)}\right)^{j} \frac{|\lambda|}{d(\lambda)}\right. \\
i=1,2,3 .
\end{array}
$$

Proof. The inequality (5.1) follows from Lemmas 2.4 and 5.1. The inequalities $(5.1)_{2}$ and $(5.1)_{3}$ can be proved analogously and we omit the proof.

For a compact subset of $\Omega, Y$. Kannai's result and ours agree. In the main theorem we assume that $\Omega$ is an $n$-box and $\Omega_{1}$ is a domain containing $\bar{\Omega}$. Letting $\widetilde{\Omega}$ be a domain containing $\bar{\Omega}$, as the assumption of the previous section, we set

$$
\sum_{(1,1)}^{\prime} a_{\alpha \beta}^{i}(x) \xi^{\alpha+\beta} \geqslant C \mu(\xi)
$$

for any $x \in \widetilde{\Omega}$, where $C$ is a constant independent of $x^{0} \in \Omega, i=1,2,3$. Let $A_{i}$ be the operator associated with $a_{i}(u, v)$, the sesquilinear form defined on $\stackrel{\circ}{H}_{m}(\widetilde{\Omega}) \times \stackrel{\circ}{H}_{m}(\widetilde{\Omega})$ by

$$
a_{i}(u, v)=\sum_{(1,1)}^{\prime} \int_{\Omega} a_{\alpha \beta}^{i-3}(x) D^{\alpha} u \overline{D^{\beta} v} d x
$$

for any $u, v \in \dot{H}_{m}(\widetilde{\Omega})$ and denote by $K_{\lambda}^{i}(x, y)$ the resolvent kernel of $A_{i}$, $i=4,5,6$. For $u \in \dot{H}_{m}(\Omega)$, we set $\tilde{u}(x)=u(x)$ for $x \in \Omega$ and $\tilde{u}(x)=0$, for $x \in \widetilde{\Omega} \backslash \Omega$. Then $\tilde{u} \in \stackrel{\circ}{H}_{m}(\Omega)$ and by this correspondence $\stackrel{\circ}{H}_{m}(\Omega)$ may be considered as a closed subspace of $\stackrel{\circ}{H}_{m}(\widetilde{\Omega})$. Recall that $\epsilon^{b}=\delta\left(x^{0}\right)=$ $\min \left\{1, \operatorname{dist}\left(x^{0}, \partial \Omega\right)\right\}$ for $x^{0} \in \Omega$. Let $S_{\lambda_{\epsilon}}^{i}$ be the operator on $H_{-m}(\widetilde{\Omega})$ to $\stackrel{\circ}{H}_{m}(\widetilde{\Omega})$ defined by

$$
S_{\lambda_{\epsilon}} f=\eta_{\epsilon}\left\{\left(A_{i-3}-\lambda\right)^{-1}(r \widetilde{f})-\left(A_{i}-\lambda\right)^{-1} f\right\}
$$

for any $f \in H_{-m}(\widetilde{\Omega})$, where $\left.\eta_{\epsilon}(x)=\eta\left(x-x^{0}\right) / \epsilon^{(1 / m)}\right)$ for $\eta \in \Lambda$ and $r f=f \mid \stackrel{\circ}{H}_{m}(\Omega)$. Of course, $S_{\lambda_{\epsilon}}^{i}$ is a bounded linear operator, $i=4,5,6$.

LEMMA 5.3. If $\epsilon^{-1}|\lambda|^{-1 / 2} \leqslant 1$ with $0<\epsilon<1$ and $|\lambda| \geqslant 1$, then for any positive integer $j$ there exists a positive constant $K_{j}$ so that for any selected $x^{0}, \epsilon$ and $\lambda$ the following estimates hold:

$$
\begin{aligned}
\left\|S_{\lambda_{\epsilon}}^{i}\right\|_{(-m, m)} & \leqslant K_{j} F(\epsilon, \lambda, j)|\lambda| d(\lambda)^{-1}, \\
\left\|S_{\lambda_{\epsilon}}^{i}\right\|_{(0, m)},\left\|S_{\lambda_{\epsilon}}^{i}\right\|_{(-m, 0)} & \leqslant K_{j} F(\epsilon, \lambda, j)|\lambda|^{1 / 2} d(\lambda)^{-1}, \\
\left\|S_{\lambda_{\epsilon}}^{i}\right\|_{(0,0)} & \leqslant K_{j} F(\epsilon, \lambda, j) d(\lambda)^{-1},
\end{aligned}
$$

where $F(\epsilon, \lambda, j)=\left(\epsilon^{-b}|\lambda|^{1-b / 2} d(\lambda)^{-1}\right)^{j}, i=4,5,6$.

PROOF. Noting that $v=\eta_{\epsilon} u \cdot=S_{\lambda_{\epsilon}}^{i} f$ with $\operatorname{supp}(v) \subset \Omega$ and $a_{i}(v, v)-\lambda(v, v)=a_{i}(v, v)-a_{i}\left(u, \eta_{\epsilon} v\right)$ the proof of the lemma will follow 
the same approach as that of Lemma 3.1. We shall omit the detail.

Lemma 5.4. For any $p>0$ and any $\lambda$ with $|\lambda| \geqslant 1$ there exists a constant $C=C(p)$, which is independent of $x^{0} \in \Omega$ and $\lambda$, such that

$$
\left|K_{\lambda}^{i-3}\left(x^{0}, x^{0}\right)-K_{\lambda}^{i}\left(x^{0}, x^{0}\right)\right| \leqslant C \frac{|\lambda|^{c}}{d(\lambda)}\left(\frac{|\lambda|^{1-b / 2}}{\delta\left(x^{0}\right) d(\lambda)}\right)^{p},
$$

$i=4,5,6$, where $c<1$.

Proof. Using the same method as in the proof of Lemma 3.2, the inequalities follow from Lemmas 2.4 and 5.3.

For the resolvent kernels of the operators with smooth coefficients Kannai has established very general results in [8]. In order to adopt his result for our purpose, we first quote from [8] the following. Let $Q=\left\{q \in R^{1} \mid q \geqslant b, q / a\right.$ is a natural number\}. The function $u \in C^{\infty}(\Omega)$ has a zero of type $q$ with respect to $m$ at a point $x^{0}$ of $\Omega$ where $q \in Q \cup\{+\infty\}$ if $u$ and all its derivatives $D^{\alpha} u$ with $|\alpha: m|<q$ vanish at $x^{0}$. If $u\left(x^{0}\right) \neq 0$ we say that $u$ has a zero of type $q=0$ at $x^{0}$.

Further, we denote by $q_{0}=q_{0}\left(x^{0}\right)$ the maximal element of $q$ in $Q \cup\{+\infty\}$; all the coefficients of $a_{\alpha \beta}(x)-a_{\alpha \beta}\left(x^{0}\right)$ for $|\alpha+\beta: m|=2$, have a zero of type $q_{0}$ at $x^{0}$, and by $q_{j}=q_{j}\left(x^{0}\right)(j>0)$ denote the maximal element of $q$ in $Q \cup\{0\} \cup\{+\infty\}$; all the coefficients of $a_{\alpha \beta}(x)$ for $|\alpha+\beta: m|$ $=2-j a$, have a zero of type $q_{j}$ at $x^{0}$. We associate with the sesquilinear form $a(u, v)$ a number $\theta\left(x^{0}\right)$ defined by

$$
\theta\left(x^{0}\right)=\min _{0 \leqslant j \leqslant 2 / a} \frac{q_{j}+j a}{q_{j}+b}
$$

where we agree that $\left(q_{j}+j a\right) /\left(q_{j}+b\right)=1$ if $q_{j}=\infty$. With the $\theta\left(x^{0}\right)$ so defined, we restate his Theorem 5.1, in somewhat restricted form, as follows (see pp. 590-591 of [8]).

Lemma 5.5 (KanNaI). Let $\Omega$ be an open subset of $R^{n}$. For any positive number $\xi$, there exists a positive constant $C=C(\xi)$, which is independent of $x^{0}$ in $\Omega$, such that

$$
\left|K_{\lambda}^{i}\left(x^{0}, x^{0}\right)-c_{0}^{i}\left(x^{0}\right)(-\lambda)^{c-1}\right| \leqslant C|\lambda|^{c-1-a / 2}, \quad i=4,5,6,
$$

for $c<1,|\lambda| \geqslant 1, d(\lambda) \geqslant|\lambda|^{1-\theta\left(x^{0}\right) b / 2+\epsilon}$, where

$$
c_{0}^{i}\left(x^{0}\right)=c_{0}\left(x^{0}\right)=(2 \pi)^{-n} \int_{R^{n}}\left(\sum_{(1,1)}^{\prime} a_{\alpha \beta}\left(x^{0}\right) \xi^{\alpha+\beta}+1\right)^{-1} d \xi
$$

where $\theta\left(x^{0}\right)$ is the number stated above.

We repeat the assertion of [8] that in the elliptic case, or in similar cases 
where $a=b$, it is possible to replace $\theta\left(x_{0}\right)$ by $1 / 2$ in general, and by 1 if the coefficients of the semiprincipal part of the kernel are constants (see [8] for more detailed accounts).

In the following we shall prove a theorem under the conditions $s-(2), s-(3)$ or $s$-(4). Since we have to apply lemmas in the previous sections in proving the theorem, we have to examine that the inequality

$$
\epsilon^{-b}|\lambda|^{1-b / 2} d(\lambda)^{-1} \leqslant 1 \text { with }|\lambda| \geqslant 1 \text { and } 0<\epsilon<1,
$$

assumed for those lemmas, holds also under the smoothness assumptions. In order to have a unified argument, we introduce three constants $C_{1}, C_{2}$ and $C_{3}$ such that $C_{1}=C_{2}=C_{3}=0$ for $s$-(2), $C_{1}=3, C_{2}=1, C_{3}=2$ for $s$-(3) and $C_{1}=4, C_{2}=C_{3}=2$ for $s$-(4) respectively. Note also that $C_{1}=C_{2}+C_{3}$ in all three cases. From

it follows that

$$
0<\theta<\frac{b\left(C_{2}+h\right)}{b\left(C_{1}+h\right)+a}
$$

$$
\frac{1}{2}\left[b \theta+\frac{\left(a+C_{3} b\right) \theta}{C_{2}+h}\right]<\frac{b}{2} \cdot \frac{b\left(C_{2}+h\right)+a+C_{3} b}{b\left(C_{1}+h\right)+a}=\frac{b}{2}
$$

which implies that

By taking

$$
\frac{\left(a+C_{3} b\right) \theta}{2\left(C_{2}+h\right)}-\frac{b}{2}<-\frac{b \theta}{2}
$$

we obtain

$$
\epsilon^{-b}|\lambda|^{-b / 2}=|\lambda|^{\left(a+C_{3} b\right) \theta / 2\left(C_{2}+h\right)-b / 2} \leqslant|\lambda|^{-b \theta / 2} \leqslant|\lambda|^{-1} d(\lambda)
$$

if $d(\lambda) \geqslant|\lambda|^{1-b \theta / 2}$ is satisfied (under the smoothness assumption). Hence the inequality is verified under the assumptions.

THEOREM 5.1. Let $\Omega$ be an $n$-box. Under the condition $s-(2), s-(3)$ or $s$-(4), the estimate

$$
\left|K_{\lambda}(x, x)-c_{0}(x)(-\lambda)^{c-1}\right| \leqslant C\left\{|\lambda|^{c-1-a / 2}+\frac{|\lambda|^{c}}{d(\lambda)}\left(\frac{|\lambda|^{1-b / 2}}{\delta(x) d(\lambda)}\right)^{p}\right\}
$$

holds for any $p>0$ and any $x \in \Omega$ provided that $d(\lambda) \geqslant|\lambda|^{1-b \theta / 2}, 0<$ $\theta<b\left(C_{2}+h\right) / b\left(C_{1}+h\right)+a$, where $c<1$ and $C$ is a constant depending only on $p$ and on $\theta$.

Proof. Under the assumption $s-(2), s-(3)$ or $s-(4)$ it follows from Lemmas $3.2,5.2$ and 5.5 that 


$$
\begin{aligned}
& \left|K_{\lambda}(x, x)-c_{0}(x)(-\lambda)^{c-1}\right| \\
& \leqslant\left|K_{\lambda}(x, x)-K_{\lambda}^{0}(x, x)\right|+\left|K_{\lambda}(x, x)-K_{\lambda}^{i}(x, x)\right| \\
& \quad+\left|K_{\lambda}^{i}(x, x)-c_{0}(x)(-\lambda)^{c-1}\right| \\
& \quad \leqslant C \frac{|\lambda|^{c}}{d(\lambda)}\left(\frac{|\lambda|^{1-b / 2}}{\delta(x) d(\lambda)}\right)^{p}+C|\lambda|^{c-1} R_{i}(\epsilon, \lambda, j)+C|\lambda|^{c-1-a / 2} .
\end{aligned}
$$

Moreover,

$$
\begin{aligned}
& C|\lambda|^{c-1}\left\{R_{i}(\epsilon, \lambda, j)+|\lambda|^{-a / 2}\right\} \\
& \begin{array}{l}
=C|\lambda|^{c-1}\left\{\epsilon^{2 b+h b-(i-1)}\left(1+\epsilon^{-h b}|\lambda|^{-a / 2}\right)\left(\frac{|\lambda|}{d(\lambda)}\right)^{2}\right. \\
\left.+|\lambda|^{-a / 2}+\left(\frac{|\lambda|^{1-b / 2}}{\epsilon^{b} d(\lambda)}\right)^{j} \frac{|\lambda|}{d(\lambda)}\right\} \\
=C|\lambda|^{c-1}\{\mathrm{I}+\mathrm{II}+\mathrm{III}\} .
\end{array}
\end{aligned}
$$

Without loss of generality we may assume $i=1$ in (5.5). It is evident from (5.2) that

$$
\frac{\left(a+C_{3} b\right) \theta}{2 b\left(C_{2}+h\right)}-\frac{1}{2}<0 .
$$

From (5.3), (5.6) and the fact that $1 / 2(h b-a) \leqslant 0$, we obtain

$$
\begin{aligned}
\epsilon^{-h b}|\lambda|^{-a / 2} & =\left(\epsilon^{-1}|\lambda|^{-1 / 2}\right)^{h b}|\lambda|^{1 / 2(h b-a)} \\
& =|\lambda|^{\left\{\left(a+C_{3} b\right) \theta / 2 b\left(C_{2}+h\right)-1 / 2\right\} h b+1 / 2(h b-a)} \leqslant C
\end{aligned}
$$

where $C$ is a positive constant, and consequently, $1+\epsilon^{-h b}|\lambda|^{-a / 2} \leqslant C$ for $|\lambda| \geqslant 1$ under the assumption $0<h \leqslant a / b$. By taking $\epsilon$ as in (5.3) and noting that $d(\lambda) \geqslant|\lambda|^{1-b \theta / 2}$, we obtain

$$
I \leqslant C^{b(2+h)}\left(\frac{|\lambda|}{d(\lambda)}\right)^{2} \leqslant C|\lambda|^{\Gamma \theta-a \theta / 2}
$$

where

$$
\begin{aligned}
\Gamma & =b+\frac{a}{2}-\frac{\left(a+C_{3} b\right)(2+h)}{2\left(C_{2}+h\right)} \\
& =\frac{2\left(C_{2}-C_{3}\right) b+\left(2-C_{3}\right) b h+\left(C_{2}-2\right) a}{2\left(C_{2}+h\right)} .
\end{aligned}
$$

Under $s-(2)\left(C_{2}=C_{3}=0\right),(5.8)$ gives

$$
\Gamma=\frac{b h-a}{h}=\frac{b}{h}\left(h-\frac{a}{b}\right) \leqslant 0 \text {. }
$$


Under $s$-(3) $\left(C_{2}=1\right.$ and $\left.C_{3}=2\right),(5.8)$ gives

$$
\Gamma=\frac{-(2 b+a)}{2(1+a)} \leqslant 0 .
$$

Under s-(4) $\left(C_{2}=C_{3}=2\right)$, (5.8) gives $\Gamma=0$. Consequently, since $\Gamma$ is nonpositive in all three cases, (5.7) implies that $I \leqslant C|\lambda|^{-a \theta / 2}$. Obviously, it follows from $\theta<1$ that

$$
\mathrm{II}=|\lambda|^{-a / 2} \leqslant|\lambda|^{-a \theta / 2} .
$$

By noting that $d(\lambda) \geqslant|\lambda|^{1-b \theta / 2}$ and taking $\epsilon$ as in (5.3), in order to achieve

$$
\text { III } \leqslant|\lambda|^{e(\theta)} \leqslant|\lambda|^{-a \theta / 2}
$$

where

$$
e(\theta)=\frac{1}{2}\left\{\frac{\left(a+C_{3} b\right) \theta}{C_{2}+h} j-b j+b \theta+b \theta j\right\}<-a \theta / 2
$$

we must impose on $\theta$ the condition

$$
\left(\frac{a+C_{3} b}{C_{2}+h} j+a+b+b j\right) \theta \leqslant b j
$$

which implies that

$$
\theta \leqslant \frac{b j}{\left(\left(a+C_{3} b\right) /\left(C_{2}+h\right)\right) j+a+b+b j}<1 .
$$

Note that the above quotient tends to $b\left(C_{2}+h\right) / b\left(C_{1}+h\right)+a$ as $j \rightarrow \infty$. Thus since I, II and III are dominated by $C|\lambda|^{-a \theta / 2}$, we obtain from (5.5) that

$$
\left|K_{\lambda}(x, x)-c_{0}(x)(-\lambda)^{c-1}\right| \leqslant C\left\{|\lambda|^{c-1-a \theta / 2}+\frac{|\lambda|^{c}}{d(\lambda)}\left(\frac{|\lambda|^{1-b / 2}}{\delta(x) d(\lambda)}\right)^{p}\right\}
$$

for $d(\lambda) \geqslant|\lambda|^{1-b \theta / 2}$ and $|\lambda| \geqslant 1$ under the condition $s-(2), s-(3)$ or $s$-(4). Hence the proof is complete.

6. Proof of main theorem. If $c<1$, then the kernel $K_{\lambda}(x, y)$ of the resolvent $(A-\lambda)^{-1}$ of $A$ is in $C(\bar{\Omega} \times \bar{\Omega})$. Since the operator $A$ is symmetric, $K_{\lambda}(x, y)=\overline{K_{\bar{\lambda}}(y, x)}$. The operator $A$ possesses a compact resolvent and the spectrum of $\boldsymbol{A}$ consists of a discrete set of eigenvalues. Let $\left\{\lambda_{j}\right\}$ and $\left\{\phi_{j}\right\}$ be the sequences of eigenvalues and corresponding eigenfunctions respectively of the orthonormal form. From Mercer's theorem,

$$
K_{\lambda}(x, y)=\sum_{j=0}^{\infty} \frac{\phi_{j}(x) \overline{\phi_{j}(y)}}{\lambda_{j}-\lambda},
$$

for any fixed $\lambda$ which belongs to the resolvent set, and the series is absolutely and uniformly convergent for any $(x, y) \in \bar{\Omega} \times \bar{\Omega}$. By using the notation 


$$
\begin{aligned}
\sigma_{x}(t) & =\sum_{\lambda_{j} \leqslant t}\left|\phi_{j}(x)\right|^{2}, \\
K_{\lambda}(x, x) & =\sum_{j=0}^{\infty} \frac{\left|\phi_{j}(x)\right|^{2}}{\lambda_{j}-\lambda}=\int_{0}^{\infty} \frac{d \sigma_{x}(t)}{t-\lambda} .
\end{aligned}
$$

We now quote two lemmas given on p. 342 of [9] as follows.

Lemma 6.1. Let $\sigma(t)$ be a nondecreasing function for $t \geqslant 0$ such that $\int_{0}^{\infty} d \sigma(t) /(1+t)<+\infty$. Suppose that

$$
f(z)=\int_{0}^{\infty} \frac{d \sigma(t)}{t-z} \text { and } I(\xi)=\frac{1}{2 \pi i} \int_{L(\xi)} f(z) d z,
$$

where $L(\xi)$ is an oriented curve in the complex plane from $\bar{\xi}$ to $\xi=t+i \tau$ not intersecting $(0, \infty)$. Then for $t, \tau>0$,

$$
|I(\xi)-(\tau / \pi) \operatorname{Re} f(\xi)-\sigma(t)+\sigma(0)| \leqslant \tau \operatorname{Im} f(\xi) .
$$

A proof of this lemma is given in [12].

LEMma 6.2. Let $\Omega$ be an $n$-box or $R^{n}$. Then there exists a positive constant $C$ such that for any $t>0$ and any $x \in \Omega, \sigma_{x}(t) \leqslant C t^{c}$ holds (where $c$ is in place of $n / 2 m$ as in [9]).

Finally we proceed in precisely the same way as in [9] to obtain the desired estimate:

$$
\left|\sigma_{x}(t)-(2 \pi)^{-n} \frac{\sin (c \pi)}{c \pi} c_{0}(x) t^{c}\right| \leqslant C t^{c-a \theta / 2} \delta(x)^{-\theta} .
$$

(6.1) holds under $s$-(2), $s$-(3) or $s$-(4). If $s$-(5) is satisfied, we can make use of that portion of the theorem of Y. Kannai (see [8] , [9]) covering the case of constant coefficients in the principal part. (6.1) is readily verified for any $\theta$ with $0<\theta<1$. Accordingly, by integrating (6.1) over $\Omega$ we obtain the asymptotic formula for $N(t)$ described in the main theorem. As far as the case $s-(1)$ is concerned, we need only investigate the asymptotic behaviour of $K_{\lambda}(x, x)$ for real $\lambda \rightarrow \infty$ and apply the tauberian theorem of Hardy and Littlewood.

Acknowledgement. The authors wish to express gratitude to Professor Hirok Tanabe for his generous counsel and encouragement during the preparation of this paper. Also they wish to thank Professor G. F. D. Duff who provided an opportunity and directed them to the common interest of research at the University of Toronto in 1968. They also wish to thank the referee for his suggestions.

\section{BIBLIOGRAPHY}

1. S. Agmon, Lectures on elliptic boundary value problems, Van Nostrand Math. Studies, no. 2, Van Nostrand, Princeton, N. J., 1965. MR 31 \#2504.

2. Asymptotic formulas with remainder estimates for eigenvalues of elliptic operators, Arch. Rational Mech. Anal. 28 (1967/68), 165-183. MR 37 \#4430. 
3. F. E. Browder, The asymptotic distribution of eigenfunctions and eigenvalues for semi-elliptic differential operators, Proc. Nat. Acad. Sci. U.S.A. 43 (1957), 270-273. MR 19, 862.

4. A. Cavallucci, Sulle proprietà differenziali delle soluzioni delle equazioni quasiellittiche relativamente a domini normali, Boll. Un. Mat. Ital. (3) 19 (1964), 465-477. MR 31 \#2501.

5. J. Friberg, Asymptotic behavior of integrals connected with spectral functions for hypoelliptic operators, Ark. Mat. 7 (1967), 283-298. MR 36 \#143.

6. E. Giusti, Equazioni quasi ellittiche e spazi $L^{p, \theta}(\Omega, \delta)$. I, Ann. Mat. Pura Appl. (4) 74 (1967), 313-354. MR 37 \#6607.

7. V. N. Gorčakov, The asymptotic behavior of a spectral function of a hypoelliptic operator of a certain class, Dokl. Akad. Nauk SSSR 152 (1963), 519-522 = Soviet Math. Dokl. 4 (1963), 1328-1332. MR 28 \#494.

8. Y. Kannai, On the asymptotic behavior of resolvent kernels, spectral functions and eigenvalues of semielliptic systems, Ann. Scuola Norm. Sup. Pisa 23 (1969), 563-634.

9. $\mathrm{K}$. Maruo and $\mathrm{H}$. Tanabe, On the asymptotic distribution of eigenvalues of operators associated with strongly elliptic sesquillinear forms, Osaka J. Math. 8 (1971), 323-345.

10. T. Matsuzawa, On quasi-elliptic boundary problems, Trans. Amer. Math. Soc. 133 (1968), 241-265. MR 37 \#600.

11. N. Nilsson, Asymptotic estimates for spectral functions connected with hypoelliptic differential operators, Math. Scand. 9 (1961), 237-251.

12. Å. Pleijel, On a theorem by P. Malliavin, Israel J. Math. 1 (1963), 166-168. MR $29 \# 5023$.

13. B. Sternin, Quasi-elliptic equations in an infinite cylinder, Dokl. Akad. Nauk SSSR 194 (1970), 1025-1028 = Soviet Math. Dokl. 11 (1970), 1347-1351. MR 42 \#6414.

14. A. Tsutsumi and C. L. Wang, On the asymptotic distribution of eigenvalues for semi-elliptic operators, Proc. Japan Acad. (to appear).

15. L. R. Volevič, Local properties of solutions of quasi-elliptic systems, Mat. Sb. 59 (101) (1962), supplement, 3-52. (Russian) MR 27 \#446.

DEPARTMENT OF MATHEMATICS, COLLEGE OF GENERAL EDUCATION, OSAKA UNIVERSITY, TOYONAKA, OSAKA 560 JAPAN

DEPARTMENT OF MATHEMATICS, UNIVERSITY OF SASKATCHEWAN, REGINA, SASKATCHEWAN, CANADA 